\title{
Probability of Success and Timelines for the Development of Vaccines for Emerging and Reemerged Viral Infectious Diseases
}

\author{
Amanda MacPherson, BSc; Nora Hutchinson, MDCM, MPhil; Oliver Schneider, MDCM; Elisabeth Oliviero, MSc; \\ Emma Feldhake, BA\&Sc; Charlotte Ouimet, BSc; Jacky Sheng; Fareed Awan, PhD; Catherine Wang; \\ Jesse Papenburg, MD, MSc; Nicole E. Basta, PhD; and Jonathan Kimmelman, PhD
}

Background: Anticipated success rates and timelines for COVID-19 vaccine development vary. Recent experience with developing and testing viral vaccine candidates can inform expectations regarding the development of safe and effective vaccines.

Objective: To estimate timelines and probabilities of success for recent vaccine candidates.

Design: ClinicalTrials.gov was searched to identify trials testing viral vaccines that had not advanced to phase 2 before 2005, and the progress of each vaccine from phase 1 through to U.S. Food and Drug Administration (FDA) licensure was tracked. Trial characteristics were doublecoded. (Registration: Open Science Framework [https://osf. io/dmuzx/]).

Setting: Trials launched between January 2005 and March 2020.

Participants: Preventive viral vaccine candidates for 23 emerging or reemerged viral infectious diseases.

Measurements: The primary end point was the probability of vaccines advancing from launch of phase 2 to FDA licensure within 10 years.

Results: In total, 606 clinical trials forming 220 distinct development trajectories (267343 enrolled participants) were identified. The probability of vaccines progressing from phase 2 to licensure within 10 years was $10.0 \%\left(95 \% \mathrm{Cl}_{\text {, }}\right.$ $2.6 \%$ to $16.9 \%$ ), with most approvals representing $\mathrm{H} 1 \mathrm{~N} 1$ or H5N1 vaccines. The average timeline from phase 2 to approval was 4.4 years (range, 6.4 weeks to 13.9 years). The probabilities of advancing from phase 1 to 2 , phase 2 to 3 , and phase 3 to licensure within the total available follow-up time were $38.2 \%(\mathrm{Cl}, 30.7 \%$ to $45.0 \%), 38.3 \%(\mathrm{Cl}, 23.1 \%$ to $50.5 \%)$, and $61.1 \%(\mathrm{Cl}, 3.7 \%$ to $84.3 \%)$, respectively.

Limitations: The study did not account for preclinical development and relied primarily on ClinicalTrials.gov and FDA resources. Success probabilities do not capture the varied reasons why vaccines fail to advance to regulatory approval.

Conclusion: Success probabilities and timelines varied widely across different vaccine types and diseases. If a SARSCoV-2 vaccine is licensed within 18 months of the start of the pandemic, it will mark an unprecedented achievement for noninfluenza viral vaccine development.

Primary Funding Source: McGill Interdisciplinary Initiative in Infection and Immunity (MI4) Emergency COVID-19 Research Funding program.

Ann Intern Med. doi:10.7326/M20-5350

Annals.org

For author, article, and disclosure information, see end of text.

This article was published at Annals.org on 24 November 2020.
D evelopment of a safe and effective vaccine for the severe acute respiratory syndrome coronavirus 2 (SARS-CoV-2) is widely regarded as a crucial public and global health goal. As of 7 October 2020, at least 48 vaccine development efforts have launched trials across 6 continents, with 30 candidates having advanced to phase 2 or phase 3 testing (1). It remains unclear how many of these candidates will withstand rigorous evaluation in field trials and when effective vaccines will be ready for deployment.

At the outset of the coronavirus disease 2019 (COVID-19) pandemic, numerous public health authorities and political leaders projected that a SARS-CoV-2 vaccine could be delivered within 12 to 18 months of laboratory testing $(2,3)$. However, projections of vaccine timelines as well as certainty of success have varied widely. Whereas some commentators anticipate that a vaccine can be easily developed within 1 year owing to immunologic characteristics of the virus, others are less sanguine (4). Moreover, vaccine development for viral diseases has occasionally encountered unexpected setbacks, including vaccine-dependent enhancement (5-7), which some commentators have suggested may play a role in the development of a SARS-CoV-2 vaccine $(8,9)$.
Although the current pandemic is unprecedented both in terms of the biological properties of SARS-CoV-2 and the strong political will and financial support being funneled into vaccine development, looking at recent vaccine development efforts may elucidate some of the factors that could affect the pace of SARS-CoV-2 vaccine development. Previous studies have used proprietary databases to estimate that $2 \%$ to $39 \%$ of vaccine candidates introduced into phase 1 trials progress to regulatory licensure (10-16). However, such studies primarily evaluated trials that predate 2009; since then, new techniques, such as next-generation sequencing and atomiclevel structural biology approaches, have emerged that may speed the pace of viral vaccine development $(17,18)$. Many such studies have included seasonal influenza vaccines or nonviral vaccines, the development of which may differ from that anticipated for COVID-19. In

See also:

Web-Only

Supplement 
this study, we estimate timelines and probabilities of success (POSs) for a recent cohort of viral vaccine candidates.

\section{Methods}

We performed a retrospective cohort study of vaccines for emerging and reemerged viral infectious diseases (EVIDs). Our primary objective was to determine the POS of vaccine development efforts, defined as the probability of a vaccine candidate advancing from the start of phase 2 to U.S. Food and Drug Administration (FDA) approval within 10 years. The start of each phase was defined as the date of first enrollment of the first trial as listed on ClinicalTrials.gov.

Our sample was created in 3 steps. First, we established a list of EVIDs. Second, we identified all prelicensure vaccine trials for each EVID registered on ClinicalTrials.gov from 1 January 2005 to 22 March 2020. Third, we organized vaccine trials into "trajectories," defined as the collection of trials testing a given vaccine candidate or regimen for a specific EVID; an example of this is shown in Appendix Figure 1 (available at Annals.org). Using FDA approval documents, we categorized trajectories as "successful" if the vaccine candidate received FDA approval at any time after 2005. We calculated the POS by using time-to-event analysis methods to account for censored data and estimated the time required to progress from earlyphase testing to approval.

\section{Data Sources}

We established a list of EVIDs by using the World Health Organization (WHO) list of epidemic and pandemic diseases (19), supplemented with EVIDs from a recent review (18). We excluded seasonal influenza because such vaccines rely on well-established platforms (20) but included pandemic ( $\mathrm{H} 1 \mathrm{~N} 1$ and avian flu) and universal flu vaccine development efforts. Universal influenza vaccines often use innovative approaches with some similarities to COVID-19 vaccine development efforts (21).

To identify vaccine development trajectories for our EVIDs of interest, we searched ClinicalTrials.gov for all prelicensure clinical trials of any phase from 2005 to present by using PubMed Medical Subject Heading synonyms for each EVID and vaccine keywords; detailed search methods are provided in the Supplement (available at Annals. org). We focused on 2005 onward because new vaccine development approaches emerged in this period (17). Trials were double-screened according to the following inclusion criteria: 1) phase 1, 2, or 3 trial; 2) preventive vaccine targeting an EVID of interest; 3 ) prelicensure trial based on trial enrollment start date; 4) testing safety, immunogenicity, or protection; and 5) monovalent vaccine candidate, for nonuniversal influenza vaccines. We excluded trials that exclusively enrolled subpopulations with comorbid conditions (such as individuals with HIV), enrolled persons who had previously participated in a trial of the same vaccine, had a pre-2005 phase 2 or 3 trial of the same vaccine candidate, or were withdrawn before

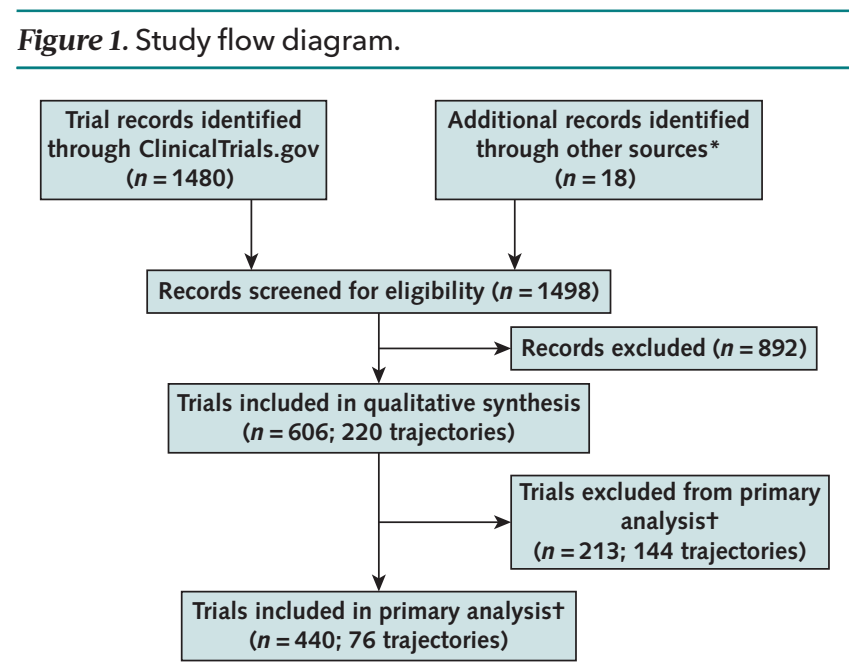

\begin{abstract}
* PubMed, World Health Organization, and U.S. Food and Drug Administration resources; these were only searched to supplement the trajectories of approved vaccine candidates or vaccines with incomplete trajectories based on ClinicalTrials.gov.

† Trajectories (and the trials they contained) were excluded from the primary analysis if they did not advance beyond phase 1 . Some trials tested multiple vaccine candidates and were thus included in multiple trajectories, so the sum of the trials included in and excluded from the primary analysis exceeds the total number of trials included in the quantitative synthesis.
\end{abstract}

recruiting any participants (Figure 1). The Supplement provides further details.

Trials were assigned to trajectories by 2 independent coders on the basis of vaccine name, type, and sponsor, and any discrepancies were adjudicated to produce the final data set. Where multiple vaccines were tested in combination, we combined vaccines into a single trajectory based on the trial that was furthest along in development (Supplement). Once trajectories had been established, we further searched PubMed, $\mathrm{WHO}$, and FDA resources to supplement our trial sample; the Supplement shows detailed search methods.

\section{Data Extraction}

Data available on ClinicalTrials.gov, including trial recruitment status, phase, enrollment, trial start and primary completion dates, were downloaded automatically. These data were manually double-extracted for trials identified through PubMed, WHO, and FDA resources (Supplement). Data not available on ClinicalTrials.gov, including vaccine type, FDA approval status, and sponsor type, were independently double-coded.

Vaccine type, approval status, and sponsor type were coded at the trajectory level. Vaccines were classified as whole-pathogen, subunit, or nucleic acid vaccines on the basis of the National Institute of Allergy and Infectious Diseases categorization (22). Split virus vaccines in influenza were classified separately (23), and trajectories that combined multiple vaccine candidates of different types were labelled as "multiple." When trial records provided insufficient information to determine the trajectory or vaccine type, we consulted pipeline 


\begin{tabular}{|c|c|c|c|c|c|}
\hline EVID & Virus Family & Trials, $n(\%)$ & Trajectories, $n$ (\%) & Enrollment, $n$ (\%)† & Approved Vaccines, $n \ddagger$ \\
\hline HIV & Retroviridae & $120(19.8)$ & $60(27.3)$ & $10612(4.0)$ & 0 \\
\hline H5N1 & Orthomyxoviridae & $117(19.3)$ & $36(16.4)$ & 50385 (18.8) & 3 \\
\hline Ebola§ & Filoviridae & $59(9.7)$ & $15(6.8)$ & $34891(13.0)$ & 1 \\
\hline H1N1 & Orthomyxoviridae & $59(9.7)$ & $15(6.8)$ & $26597(9.9)$ & 5 \\
\hline H7N9 & Orthomyxoviridae & $30(5.0)$ & $15(6.8)$ & $7313(2.7)$ & 0 \\
\hline Zika & Flaviviridae & $19(3.1)$ & $14(6.4)$ & $3609(1.3)$ & 0 \\
\hline Universal flu & Orthomyxoviridae & $26(4.3)$ & $10(4.5)$ & $14538(5.4)$ & 0 \\
\hline EV71 & Picornaviridae & $22(3.6)$ & $8(3.6)$ & 39507 (14.8) & 0 \\
\hline Dengue & Flaviviridae & $88(14.5)$ & $7(3.2)$ & $73697(27.5)$ & 1 \\
\hline West Nile & Flaviviridae & $9(1.5)$ & $6(2.7)$ & $918(0.3)$ & 0 \\
\hline Chikungunya & Togaviridae & $12(2.0)$ & $5(2.3)$ & $1581(0.6)$ & 0 \\
\hline $\mathrm{H} 3 \mathrm{~N} 2 \mathrm{v}$ & Orthomyxoviridae & $7(1.2)$ & $4(1.8)$ & $1235(0.5)$ & 0 \\
\hline Hepatitis C & Flaviviridae & $6(1.0)$ & $4(1.8)$ & $622(0.2)$ & 0 \\
\hline Marburg§ & Filoviridae & $5(0.8)$ & $4(1.8)$ & $305(0.1)$ & 0 \\
\hline MERS & Coronaviridae & $8(1.3)$ & $4(1.8)$ & $101(0)$ & 0 \\
\hline Smallpox & Poxviridae & $7(1.2)$ & $3(1.4)$ & $1248(0.5)$ & 2 \\
\hline Yellow fever & Flaviviridae & $3(0.5)$ & $3(1.4)$ & $152(0.1)$ & 0 \\
\hline Hantavirus & Hantaviridae & $4(0.7)$ & $2(0.9)$ & $79(0)$ & 0 \\
\hline $\mathrm{CCHF}$ & Bunyaviridae & $1(0.2)$ & $1(0.5)$ & $60(0)$ & 0 \\
\hline H10N8 & Orthomyxoviridae & $1(0.2)$ & $1(0.5)$ & $201(0.1)$ & 0 \\
\hline Lassa & Arenaviridae & $1(0.2)$ & $1(0.5)$ & $0(0)$ & 0 \\
\hline Nipah & Paramyxoviridae & $1(0.2)$ & $1(0.5)$ & $0(0)$ & 0 \\
\hline Rift Valley & Bunyaviridae & $1(0.2)$ & $1(0.5)$ & $20(0)$ & 0 \\
\hline
\end{tabular}

$\mathrm{CCHF}=$ Crimean-Congo hemorrhagic fever; EV71 = enterovirus 71; EVID = emerging or reemerged viral infectious disease; MERS = Middle East respiratory syndrome.

* Four EVIDs did not have any eligible trials (Hendra virus, adenovirus 14, severe acute respiratory syndrome, and monkeypox).

† Enrollment numbers include only patients in trials that had "actual"; enrollment listed on ClinicalTrials.gov.

¥ Number of vaccines approved by the U.S. Food and Drug Administration since 2005. One H1N1 vaccine (Influenza A [H1N1] 2009 Monovalent Vaccine; Medlmmune LLC) and the 2 smallpox vaccines (Jynneos; Smallpox and Monkeypox Vaccine, Live, Non-Replicating; Bavarian Nordic A/S and ACAM2000; Smallpox [Vaccinia] Vaccine, Live; Emergent Product Development Gaithersburg Inc.) had no eligible trials and were excluded from the analysis.

$\S$ Two trajectories overlap between Marburg and Ebola.

reviews and press releases to resolve uncertainties. The FDA approval status was determined on the basis of the date of first approval for each vaccine. Trajectory sponsorship was based on trial records for the earliest 2 trials per trajectory; the Supplement provides details on sponsor classification.

\section{Data Synthesis}

The primary outcome was the POS from launch of phase 2 within 10 years. We anchored our primary outcome at phase 2 rather than phase 1 because sponsors are not legally required to register phase 1 trials on ClinicalTrials.gov (24) and publication bias would make it almost impossible to reliably identify all vaccine candidates that produced negative results in phase 1 trials (25). Trajectories that did not advance beyond phase 1 were not included in the primary analysis. The follow-up time of 10 years from the enrollment start date was selected because it is in line with prior estimates of vaccine development time $(15,16)$.

To evaluate the POS, we used time-to-event analysis, which accounts for the fact that newer vaccine development efforts have less follow-up time than older ones. We report the Kaplan-Meier estimate at 10 years, along with $95 \% \mathrm{Cls}$ based on the log-transformation of survival.

As a secondary outcome, we looked at the probabilities of phase transition events from the enrollment start date of each phase to the enrollment start date of the subsequent phase. The probabilities of transitioning from one phase to the next were calculated for phase 1 to phase 2, phase 2 to phase 3 , phase 3 to approval by using the time-to-event analysis method described above (Supplement). We report the transition probabilities at the total available follow-up time of 15.5 years, 15.0 years, and 14.1 years from phase 1 , phase 2, and phase 3 , respectively. We also looked at the POS from initiation of phase 1 within 12 years to include more of our data set; this can be interpreted as an upper limit on the POS from phase 1, given that our search may not have captured all vaccine candidates that did not advance past phase 1 .

As a sensitivity analysis to account for the fact that vaccine candidates may fail to advance to regulatory 
approval owing to an outbreak quelling (which would impede trial accrual and lower vaccine demand), we reanalyzed our primary outcome of POS from phase 2 to approval, including only those vaccine candidates for which termination of trial activity did not coincide with outbreak quelling. We defined a quelling as a 12-month period without active cases listed in the WHO Outbreak Archive, with the exception of endemic diseases, including HIV, hepatitis C, and universal influenza, and excluded vaccines from the sensitivity analysis if a quelling followed the primary completion date of the last active trial in the trajectory. In addition, we report the total proportion of "unsuccessful" vaccines whose terminations coincided with a quelling and the proportion of trajectories that persisted despite a quelling.

We assessed whether there were differences in trajectory success probabilities stratified by vaccine type and indication (influenza versus noninfluenza). All analyses were descriptive. Analyses were performed with R, version 3.5.0, and time-to-event analyses were performed by using the survival package on 22 June 2020 (26). Outcomes were prespecified before extraction, and the study was prospectively registered on Open Science Framework (https://osf.io/dmuzx/); the Supplement provides an explanation of differences from the registration record. The full data set and statistical code is available on Open Science Framework (https://osf.io/ed6ar/).

\section{Role of the Funding Source}

The study was funded by the McGill Interdisciplinary Initiative in Infection and Immunity (MI4) Emergency COVID-19 Research Funding program. The funding source had no role in the design or execution of this study or in the decision to submit the manuscript for publication.

\section{Results}

Of the 27 EVIDs included in our initial search, we identified at least 1 eligible trial on ClinicalTrials.gov for 23 (Table 1). Our trial search captured 606 eligible trials forming 220 trajectories and enrolling 267343 volunteers (Table 2). Of the 220 vaccine trajectories, 9 advanced to FDA approval, 7 of which were approved within 10 years of phase 2 start, and 76 advanced beyond phase 1 and were included in the primary analysis.

The timeline from the launch of the first phase 2 trial to FDA approval ranged from 6.4 weeks (Influenza A [H1N1] 2009 Monovalent Vaccine [Sanofi Pasteur, Inc.] and Influenza A [H1N1] 2009 Monovalent Vaccine [Novartis Vaccines and Diagnostics Ltd.]) to 13.9 years (Audenz Influenza A [H5N1] Monovalent Vaccine, Adjuvanted [Seqirus Inc.]), with an average of 4.4 years (Table 3).

\section{Vaccine Development POS}

The POS from phase 2 launch was 10.0\% $(95 \% \mathrm{Cl}$, $2.6 \%$ to $16.9 \%$ ) within 10 years (Figure 2 ). The 7 vaccines that received approval within 10 years were Ervebo
Table 2. Trial and Trajectory Characteristics

\begin{tabular}{ll}
\hline Characteristic & Value \\
\hline Trials & \\
\hline Total trials, $n$ & 606 \\
Median (range) trials per EVID, $n$ & $8(1-120)$ \\
Median (range) trials per trajectory, $n$ & $1(1-30)$ \\
Trial phase, $n$ (\%) & \\
Phase 1 & $319(52.6)$ \\
Phase $1 / 2^{*}$ & $59(9.7)$ \\
Phase 2 & $154(25.4)$ \\
Phase $2 / 3^{*}$ & $5(0.8)$ \\
Phase 3 & $69(11.4)$
\end{tabular}

Trajectories

Total trajectories, $n \quad 220$

Median (range) trajectories per EVID, $n \quad 4$ (1-60)

Patients

Total, $n \dagger \quad 267343$

Median (range) patients per EVID, $n \quad 1248$ (20-73 697)

Median (range) patients per EVID, $n \quad 147$ (1-44 500)

Vaccine type, $n$ (\%)

Whole-pathogen $\quad 53(24.1)$

Split virus $14(6.4)$

Subunit $\quad 40(18.2)$

Nucleic acid $103(46.8)$

Multiple $10(4.5)$

Highest phase reached, $n$ (\%)

Phase $1 \quad 144(65.5)$

Phase $2 \quad 54(24.5)$

Phase 32 (10.0)

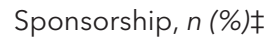

Large companies $\quad 35$ (15.9)

Small companies 90 (40.9)

Government $129(58.6)$

Foundation $19(8.6)$

Other 79 (35.9)

EVID = emerging or reemerged viral infectious disease.

* 51 of 59 phase $1 / 2$ trials and 4 of 5 phase $2 / 3$ trials were considered as phase 2 and phase 3 trials, respectively, on the basis of completion status and enrollment.

† Enrollment in trials that overlapped between EVIDs or trajectories was only counted once. Enrollment was not counted if it was listed as "anticipated" on ClinicalTrials.gov.

$\ddagger$ Sponsorship was based on the earliest 2 trials per trajectory. "Large company" was defined as a pharmaceutical company listed among the top 10 companies by sales in the year of trial start on ContractPharma; all other companies were considered to be small companies. The percentage sum exceeds $100 \%$ because many trajectories had multiple sponsors.

(Ebola Zaire Vaccine, Live [Merck Sharp \& Dohme Corp.]) and 6 pandemic influenza vaccines. Dengvaxia (Dengue Tetravalent Vaccine, Live [Sanofi Pasteur Inc.]) and Audenz were approved 12.7 and 13.9 years from initiation of phase 2, respectively. The POS from phase 1 was $7.1 \%(\mathrm{Cl}, 1.3 \%$ to $12.5 \%)$ within 12 years. 


\section{Phase Transition Probabilities}

The probabilities that development trajectories would advance from phase 1 to 2 , phase 2 to 3 , and phase 3 to approval within the total follow-up time were $38.2 \%(\mathrm{Cl}, 30.7 \%$ to $45.0 \%), 38.3 \%(\mathrm{Cl}, 23.1 \%$ to $50.5 \%)$, and $61.1 \%(\mathrm{Cl}, 3.7 \%$ to $84.3 \%)$, respectively (Appendix Table 1, available at Annals.org). Appendix Figure 2 (available at Annals.org) shows Kaplan-Meier curves.

\section{Variation in POS by Indication and Vaccine Type}

Excluding pandemic influenza trajectories, which largely build on an established vaccine development platform, the POS from phase 2 to approval was 3.2\% $(\mathrm{Cl}, 0.0 \%$ to $9.2 \%)$ within 10 years. Of all vaccine types, the only categories that had an FDA approval within 10 years were split virus and nucleic acid vaccines, with $56.4 \%(\mathrm{Cl}, 12.6 \%$ to $78.2 \%)$ of split virus vaccines and $5.9 \%(\mathrm{Cl}, 0.0 \%$ to $16.4 \%)$ of nucleic acid vaccines progressing from phase 2 to FDA licensure within 10 years (Appendix Table 2, available at Annals.org).

\section{Sensitivity Analyses}

Of the 211 trajectories that did not advance to FDA approval and of the 67 of these included in our primary analysis, $23.2 \%$ (49 of 211) and 29.9\% (20 of 67), respectively, terminated during a quelling of $\mathrm{WHO}$ reported outbreak activity. After excluding these 20 trajectories, the POS from phase 2 was $13.5 \%(\mathrm{Cl}, 3.6 \%$ to $22.5 \%)$ overall and $5.0 \%(\mathrm{Cl}, 0.0 \%$ to $14.1 \%)$ for noninfluenza vaccines. Vaccine development efforts often persevered in spite of outbreak quelling. There were 77 vaccine development trajectories with at least 1 quelling of disease activity from trajectory start to within 1 year after trajectory end. Of these, 54 (70.1\%) continued with further development through at least 1 quelling, 3 of which were approved (Dengvaxia, Ervebo, and Audenz).

\section{Discussion}

To our knowledge, this analysis provides the first estimate of preventive EVID vaccine development success rates from phase 2 to licensure by using publicly available data $(27,28)$. Our findings suggest that approximately 1 in 10 EVID vaccines that reached phase 2 since 2005 advanced to FDA licensure within 10 years, whereas 1 in 30 advanced when influenza vaccines were excluded. Of the 23 EVIDs in our sample, 4 diseases had at least 1 vaccine candidate that obtained an approval (dengue, Ebola, H1N1, and H5N1). However, FDA approval is an imperfect proxy for successful vaccine

Table 3. Characteristics of Approved Vaccines

\begin{tabular}{|c|c|c|c|c|c|}
\hline Vaccine & Manufacturer & Vaccine Type & Phase 2 Start $^{*}$ & Approval Date & Years to Approval \\
\hline $\begin{array}{l}\text { Influenza A (H1N1) } \\
2009 \text { Monovalent } \\
\text { Vaccine }\end{array}$ & $\begin{array}{l}\text { Novartis Vaccines and } \\
\text { Diagnostics Ltd. }\end{array}$ & Split virus & 1 August 2009 & 15 September 2009 & 0.1 \\
\hline $\begin{array}{l}\text { Influenza A (H1N1) } \\
2009 \text { Monovalent } \\
\text { Vaccine }^{a}\end{array}$ & CSL Limited & Split virus & 1 July 2009 & 15 September 2009 & 0.2 \\
\hline $\begin{array}{l}\text { Influenza A (H1N1) } \\
2009 \text { Monovalent } \\
\text { Vaccine }\end{array}$ & ID Biomedical Corp. & Split virus & 11 August 2009 & 10 November 2009 & 0.2 \\
\hline $\begin{array}{l}\text { Influenza Virus } \\
\text { Vaccine, H5N1 (for } \\
\text { National } \\
\text { Stockpile) }\end{array}$ & Sanofi Pasteur, Inc. & Split virus & 21 June 2005 & 17 April 2007 & 1.8 \\
\hline $\begin{array}{l}\text { Dengue Tetravalent } \\
\text { Vaccine, Live } \\
\text { (Dengvaxia) }\end{array}$ & Sanofi Pasteur, Inc. & Whole-pathogen & 1 August 2006 & 1 May 2019 & 12.7 \\
\hline $\begin{array}{l}\text { Influenza A (H5N1) } \\
\text { Monovalent } \\
\text { Vaccine, } \\
\text { Adjuvanted } \\
\text { (Audenz) }\end{array}$ & Seqirus Inc. & Subunit & 1 March 2006 & 31 January 2020 & 13.9 \\
\hline
\end{tabular}

* Enrollment launch date of the first phase 2 trial. If the first phase 2 was a phase $1 / 2$ trial, phase 2 start was considered to be one third of the way through the total phase 1/2 trial duration. This was the case for Ervebo, Audenz, and the Sanofi H5N1 vaccine.

† Approved without a phase 3 trial.

¥ This vaccine had a phase 3 trial that preceded the first phase 2 trial; the date reflects the start of the phase 3 trial. 
Figure 2. Kaplan-Meier curve for probability of FDA approval from the time of launch of the phase 2 trial.

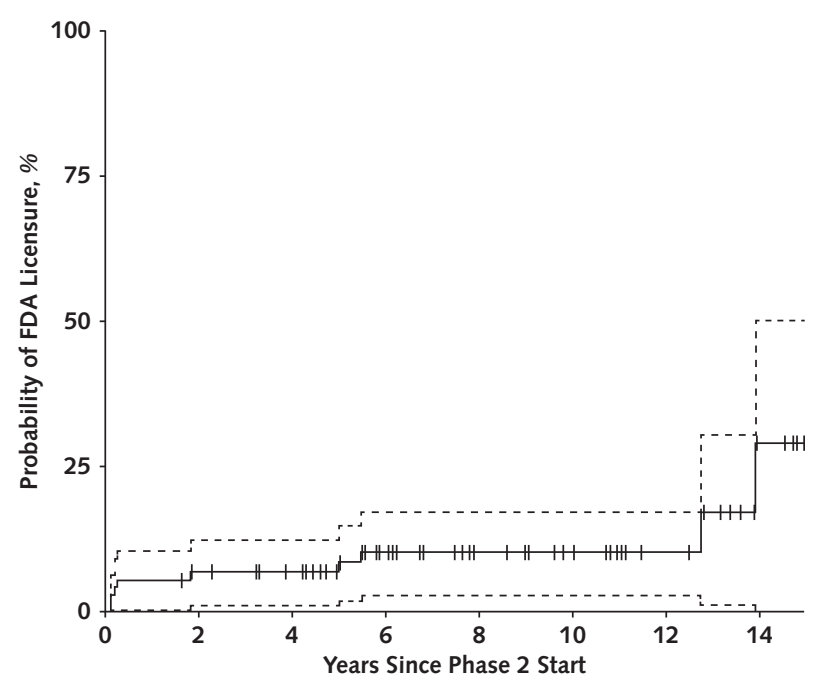

Vaccines with potential for approval, $n$

$\begin{array}{llllllll}76 & 68 & 63 & 47 & 36 & 30 & 17 & 5\end{array}$

Vertical tick marks indicate censored vaccine trajectories. Dotted lines indicate $95 \% \mathrm{Cls}$. FDA = U.S. Food and Drug Administration.

development and may not equate with public health impact. For instance, Dengvaxia is only $61 \%$ efficacious in endemic settings and is associated with enhanced disease $(6,29)$. In addition, the effect of an approval may be limited by the quelling of the initial epidemic, as in the 2014-2016 Ebola epidemic in West Africa (30).

Our results also highlight wide variation in timelines, development activities, and success rates in vaccine development across different EVIDs (Figure 3). H1N1 and H5N1 influenza vaccines accounted for most approved vaccines in our sample and also had the shortest clinical development times (with the exception of Audenz, which took 13.9 years). However, the rapid development of $\mathrm{H} 1 \mathrm{~N} 1$ and $\mathrm{H} 5 \mathrm{~N} 1$ vaccines is probably attributable to existing seasonal influenza research and platforms (20). Vaccine development efforts for SARS-CoV-2 are building on a much more limited base of research and vaccine candidates, including those developed using less wellestablished platforms. In addition, increased trial or trajectory volume was not necessarily associated with successful licensure. Vaccines designed to prevent HIV accounted for $20 \%$ of trials and almost $30 \%$ of trajectories but had only 1 vaccine regimen that reached phase 3 since 2005 and no approvals. In our sample, the most rapid successful noninfluenza vaccine development timeline was for the Ebola recombinant vector vaccine, Ervebo (5.0 years overall from phase 2 to approval), which also is the only approval of a nucleic acid vaccine to date.

If COVID-19 vaccine development were to follow historic timelines, progress would be insufficient, given the urgency of controlling the pandemic (31). Our results should be viewed as a systematic assessment of historically observed success rates and timelines of viral vaccine development rather than necessarily foreshadowing the outcome of COVID-19 efforts. Nevertheless, our findings point to challenges that SARS-CoV-2 vaccine development will need to overcome and highlight the need for new paradigms that can facilitate the development of vaccines "at pandemic speed" (31).

As of 7 October 2020, at least 48 SARS-CoV-2 vaccine candidates had entered clinical testing, 19 of which are in phase 2 clinical trials and 11 of which are in phase 3 (1). Current SARS-CoV-2 vaccine development efforts differ from those for the EVIDs in our study in several ways. First, many outbreaks of EVIDs in our sample, such as SARS, ended before vaccines were developed, which is less likely to be the case for COVID-19. On the other hand, for some EVIDs in our sample (such as HIV and universal influenza), disease activity and public interest remained high throughout the study period. In addition, our sensitivity analysis indicated that the overall success probability does not change substantially after vaccines for which trajectory discontinuation coincided with a quelling in WHO-reported outbreak activity are excluded. Second, many SARS-CoV-2 vaccine candidates have condensed or truncated the usual basic science research and preclinical steps and are conducting traditionally distinct clinical trial phases in parallel. This could accelerate timelines, though careful attention will be needed to ensure that sufficient evidence is generated for advancement of candidates to further testing and licensure. Third, the global collaboration facilitated by private-public partnerships and organizations, such as the Coalition for Epidemic Preparedness Innovations, to expedite development timelines is unprecedented $(32,33)$. Finally, with almost 50 COVID-19 vaccine candidates in the clinical pipeline, the volume of candidates is already similar to the volume of experimental vaccine platforms that HIV vaccine efforts produced over the past 15 years.

The above dynamics nevertheless contend with uncertainty regarding the biology of SARS-CoV-2. SARSCoV-2 is the world's third encounter with a novel coronavirus that has pandemic potential. Although research on SARS-CoV and MERS-CoV provided a springboard for SARS-CoV-2 $(34,35)$, no coronavirus vaccines have yet been evaluated in large field studies, and the possibility of vaccine-dependent enhancement or other adverse safety events provide grounds for caution $(8,9)$. In addition, some companies are pursuing novel approaches, such as recombinant vectors and proteins and mRNA and DNA vaccines $(31,36)$. No mRNA or DNA vaccines have previously been licensed. However, many other SARS-CoV-2 vaccine candidates are being developed by using previously successful technologies, such as inactivated vaccines. Our findings underscore the importance of pursuing both conventional and novel platforms to maximize chances of success, especially given that for sufficient doses to be available to vaccinate various atrisk populations, multiple vaccines are likely to be needed worldwide. 
Figure 3. Vaccine trajectories for EVIDs.

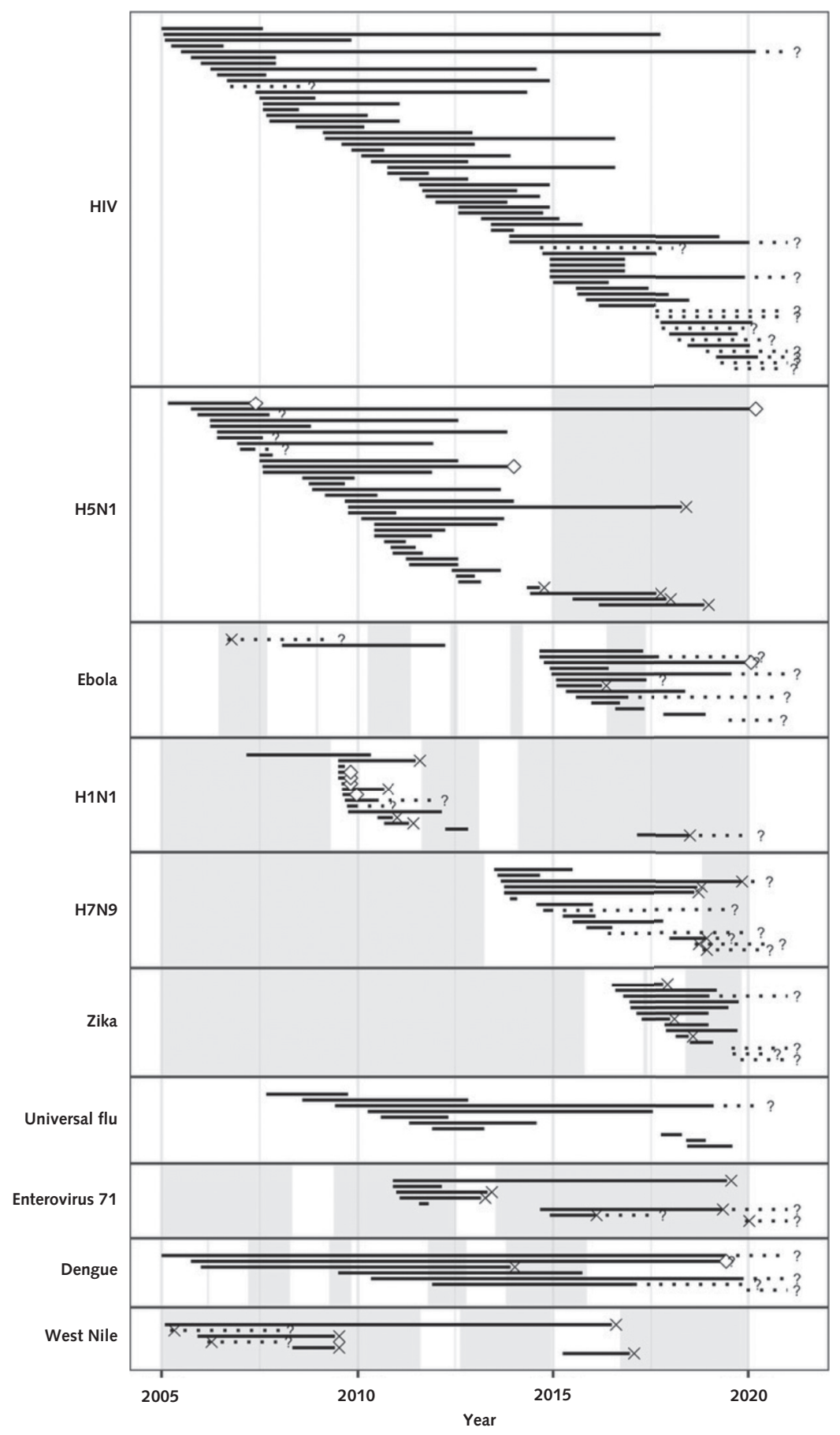

Each horizontal bar represents a single trajectory composed of 1 or more trials, beginning at the start date of the earliest trial of any phase since 2005 and ending at the primary completion date of the latest trial or the date of U.S. Food and Drug Administration (FDA) approval. If the latest primary completion date was marked as "anticipated" on ClinicalTrials.gov or the trial status was not marked as "completed," "active, not recruiting," or "terminated," the confirmed trajectory duration is indicated by a solid bar, and the interval between the latest confirmed date and the latest possible date is indicated by a segmented bar. Lines are censored at 2021 for trials with anticipated completion dates later than 2021. Only EVIDs with more than 5 trajectories are shown. Trajectories are ordered by start date within each EVID. Open diamonds indicate vaccine candidates that received FDA approval; X's indicate the latest confirmed primary completion date for a trajectory that coincides with a quelling in World Health Organization-reported outbreak activity. Question marks indicate trajectories for which the end date could not be confirmed. Gray shading indicates periods of 1 year or more between 2005 and 2020 where there was no World Health Organization-reported outbreak activity in the preceding year. EVID = emerging and reemerged viral infectious disease. 
Our study has limitations. First, we did not account for time invested in preclinical development or manufacturing and distribution, which represent substantial portions of the development timeline for new vaccines (3). Second, we relied primarily on ClinicalTrials.gov and FDA resources to create our trial sample and identify approvals. We may be missing phase 1 trials, which are not required to be registered on ClinicalTrials.gov (24); for this reason, we anchored our primary outcome at phase 2. Also, our analysis only captured approvals by the FDA. At least 4 nonU.S. approvals of vaccines in our data set were not captured in our analysis (2 enterovirus 71 approvals in China and $1 \mathrm{H} 1 \mathrm{~N} 1$ influenza approval each in South Korea and India) $(37,38)$, in addition to H5N1 influenza vaccines licensed by the European Medicines Agency (39). Third, our estimates were heavily influenced by influenza and HIV vaccine development efforts. Finally, our analysis did not enable assessment of whether trajectories failed owing to safety, efficacy, decline in disease activity, or commercial reasons. Disentangling explanations for vaccine development failure is likely to be challenging given the many contributing factors. For example, low efficacy may render safety concerns more salient. In addition, while we looked at quelling of disease activity as a possible explanation for trajectory discontinuation, this is only one of many possible causes, and we are not aware of any systematic attempts to assess reasons for vaccine development failure. Future studies are needed to elucidate the factors contributing to the failure of vaccine candidates, as well as to probe the concordance between the outcomes of early and late phase trials.

Low success rates and long timelines for recent viral vaccine development underscore the extensive challenges of developing a safe and effective SARSCoV-2 vaccine within 18 months of its emergence. Nevertheless, new collaborative paradigms of vaccine development and an unprecedented number and diversity of development efforts offer some prospect that such challenges may be overcome. Although it is difficult to predict which platforms or strategies will prove most efficient from a timeline perspective, maintaining active research programs on various emerging infectious diseases and developing platforms that can be easily mobilized for emerging threats are likely to be critical to reducing vaccine development timelines in the future.

From Biomedical Ethics Unit, McGill University, Montreal, Quebec, Canada (A.M., N.H., O.S., E.O., E.F., C.O., J.S., F.A., C. W., J.K.); McGill University, Montreal, Quebec, Canada (J.P., N. E.B.)

Acknowledgment: The authors thank Dr. Patrick Kane for his input in developing the protocol and for his feedback on the manuscript.
Financial Support: By the McGill Interdisciplinary Initiative in Infection and Immunity (MI4) Emergency COVID-19 Research Funding program.

Disclosures: Disclosures can be viewed at www.acponline.org/ authors/icmje/ConflictOfInterestForms.do?msNum=M20-5350.

Reproducible Research Statement: Study protocol, statistical code, and data set: Available on Open Science Framework (https://osf.io/dmuzx).

Corresponding Author: Jonathan Kimmelman, PhD, Biomedical Ethics Unit, Department of Social Studies of Medicine, McGill University, 3647 Peel Street, Montreal, OC H3A 1X1, Canada; e-mail, jonathan.kimmelman@mcgill.ca.

Current author addresses and author contributions are available at Annals.org.

\section{References}

1. McGill COVID19 Vaccine Tracker Team. COVID19 vaccine tracker. October 2020. Accessed at http://covid19.trackvaccines. org on 14 October 2020.

2. Cohen J. Unveiling 'Warp Speed,' the White House's Americafirst push for a coronavirus vaccine. Science. 12 May 2020. Accessed at www.sciencemag.org/news/2020/05/unveiling-warpspeed-white-house-s-america-first-push-coronavirus-vaccine on 13 May 2020.

3. Thompson SA. How long will a vaccine really take? New York Times. 30 April 2020. Accessed atwww.nytimes.com/interactive/ 2020/04/30/opinion/coronavirus-covid-vaccine.html on 30 April 2020.

4. Kane PB, Moyer $H$, MacPherson A, et al. Expert forecasts of COVID-19 vaccine development timelines [Letter]. J Gen Intern Med. 2020. [PMID: 32989715] doi:10.1007/s11606-020-06244-9

5. Sekaly RP. The failed HIV Merck vaccine study: a step back or a launching point for future vaccine development. J Exp Med. 2008;205:7-12. [PMID: 18195078] doi:10.1084/jem.20072681 6. Halstead SB. Dengvaxia sensitizes seronegatives to vaccine enhanced disease regardless of age. Vaccine. 2017;35:63556358. [PMID: 29029938] doi:10.1016/j.vaccine.2017.09.089

7. Acosta PL, Caballero MT, Polack FP. Brief history and characterization of enhanced respiratory syncytial virus disease. Clin Vaccine Immunol. 2015;23:189-95. [PMID: 26677198] doi:10.1128/CVI. 00609-15

8. Iwasaki A, Yang Y. The potential danger of suboptimal antibody responses in COVID-19. Nat Rev Immunol. 2020;20:339341. [PMID: 32317716] doi:10.1038/s41577-020-0321-6

9. ambert PH, Ambrosino DM, Andersen SR, et al. Consensus summary report for CEPI/BC March 12-13, 2020 meeting: assessment of risk of disease enhancement with COVID-19 vaccines. Vaccine. 2020;38:4783-4791. [PMID: 32507409] doi:10.1016/j.vaccine.2020 .05 .064

10. Wong $\mathrm{CH}$, Siah KW, Lo AW. Estimation of clinical trial success rates and related parameters. Biostatistics. 2019;20:273-286. [PMID: 29394327] doi:10.1093/biostatistics/kxx069

11. Hay M, Thomas DW, Craighead JL, et al. Clinical development success rates for investigational drugs. Nat Biotechnol. 2014;32:4051. [PMID: 24406927] doi:10.1038/nbt.2786

12. Thomas D, Burns J, Audette J, et al. Clinical development success rates 2006-2015. BIO, BioMedTracker, and Amplion. June 2016. Accessed atwww.bio.org/sites/default/files/legacy/bioorg/ docs/Clinical\%20Development\%20Success\%20 Rates\%202006- 
2015\%20-\%20BIO,\%20Biomedtracker,\%20Amplion\%202016.pdf on 17 June 2020.

13. Davis MM, Butchart AT, Wheeler JR, et al. Failure-to-success ratios, transition probabilities and phase lengths for prophylactic vaccines versus other pharmaceuticals in the development pipeline. Vaccine. 2011;29:9414-6. [PMID: 22001883] doi:10.1016/j. vaccine.2011.09.128

14. World Health Organization; Norwegian Institute of Public Health. An R\&D blueprint for action to prevent epidemics: funding $\&$ coordination models for preparedness and response. World Health Organization; May 2016.

15. Struck MM. Vaccine R\&D success rates and development times. Nat Biotechnol. 1996;14:591-3. [PMID: 9630948]

16. Pronker ES, Weenen TC, Commandeur $\mathrm{H}$, et al. Risk in vaccine research and development quantified. PLoS One. 2013;8:e57755. [PMID: 23526951] doi:10.1371/journal.pone.0057755

17. Graham BS, Sullivan NJ. Emerging viral diseases from a vaccinology perspective: preparing for the next pandemic. Nat Immunol. 2018;19:20-28. [PMID: 29199281] doi:10.1038/s41590017-0007-9

18. Marston HD, Folkers GK, Morens DM, et al. Emerging viral diseases: confronting threats with new technologies. Sci Transl Med. 2014;6:253ps10. [PMID: 25210060] doi:10.1126/scitranslmed. 3009872

19. World Health Organization. Pandemic \& epidemic diseases. 2014. Accessed atwww.who.int/csr/disease/WHO_PED_flyer_2014. PDF?ua=1 on 8 May 2020.

20. Weir JP, Gruber MF. An overview of the regulation of influenza vaccines in the United States. Influenza Other Respir Viruses. ;2016;10:354-60. [PMID: 27426005] doi:10.1111/irv.12383

21. Wei CJ, Crank MC, Shiver J, et al. Next-generation influenza vaccines: opportunities and challenges. Nat Rev Drug Discov. ;2020;19:239-252. [PMID: 32060419] doi:10.1038/s41573-0190056-x

22. National Institute of Allergy and Infectious Diseases; National Institutes of Health. Vaccine types. 1 July 2019. Accessed atwww. niaid.nih.gov/research/vaccine-types on 21 March 2020.

23. World Health Organization. Influenza vaccines. 19 June 2019. Accessed atwww.who.int/biologicals/vaccines/influenza/en on 25 June 2020.

24. ClinicalTrials.gov. FDAAA 801 and the Final Rule. August 2020. Accessed athttps://clinicaltrials.gov/ct2/manage-recs/fdaaa on 14 October 2020.

25. Manzoli L, Flacco ME, D'Addario M, et al. Non-publication and delayed publication of randomized trials on vaccines: survey. BMJ. 2014;348:g3058. [PMID: 24838102] doi:10.1136/bmj. g3058
26. Therneau T. A package for survival analysis in R. 2020. R Project. Accessed athttps://CRAN.R-project.org/package=survival on 16 June 2020.

27. Pronker ES, Weenen TC, Commandeur HR, et al. The gold industry standard for risk and cost of drug and vaccine development revisited. Vaccine. 2011;29:5846-9. [PMID: 21722688] doi:10.1016/ j.vaccine.2011.06.051

28. Chit A, Parker J, Halperin SA, et al. Toward more specific and transparent research and development costs: the case of seasonal influenza vaccines. Vaccine. 2014;32:3336-40. [PMID: 23830976] doi:10.1016/j.vaccine.2013.06.055

29. Villar L, Dayan GH, Arredondo-García JL, et al; CYD15 Study Group. Efficacy of a tetravalent dengue vaccine in children in Latin America. N Engl J Med. 2015;372:113-23. [PMID: 25365753] doi10.1056/NEJMoa1411037

30. Venkatraman N, Silman D, Folegatti PM, et al. Vaccines against Ebola virus. Vaccine. 2018;36:5454-5459. [PMID:28780120] doi:10.1016/j.vaccine.2017.07.054

31. Lurie N, Saville M, Hatchett $R$, et al. Developing covid-19 vaccines at pandemic speed. N Engl J Med. 2020;382:19691973. [PMID: 32227757] doi:10.1056/NEJMp2005630

32. Corey L, Mascola JR, Fauci AS, et al. A strategic approach to COVID-19 vaccine R\&D. Science. 2020;368:948-950. [PMID: 32393526] doi:10.1126/science.abc5312

33. Apuzzo M, Kirkpatrick DD. Covid-19 changed how the world does science, together. New York Times. 1 April 2020. Accessed at www.nytimes.com/2020/04/01/world/europe/coronavirus-scienceresearch-cooperation.html on 17 June 2020.

34. Ahmed SF, Quadeer AA, McKay MR. Preliminary identification of potential vaccine targets for the COVID-19 coronavirus (SARS-CoV-2) based on SARS-CoV immunological studies. Viruses. 2020; 12. [PMID: 32106567 ] doi:10.3390/v12030254

35. Padron-Regalado E. Vaccines for SARS-CoV-2: lessons from other coronavirus strains. Infect Dis Ther. 2020:1-20. [PMID: 32328406] doi:10.1007/s40121-020-00300-x

36. Le TT, Andreadakis Z, Kumar A, et al. The COVID-19 vaccine development landscape. Nat Rev Drug Discov. 2020;19:305-6. doi:10.1038/d41573-020-00073-5

37. Reed Z, Cardosa MJ. Status of research and development of vaccines for enterovirus 71. Vaccine. 2016;34:2967-2970. [PMID: 26973065] doi:10.1016/j.vaccine.2016.02.077

38. World Health Organization. WHO prequalified vaccines. 11 September 2020. Accessed athttps://extranet.who.int/gavi/PQ_ Web on 11 September 2020.

39. European Medicines Agency. Vaccines for pandemic influenza. 2018. Accessed atwww.ema.europa.eu/en/humanregulatory/overview/public-health-threats/pandemic-influenza/ vaccines-pandemic-influenza on 4 June 2020. 
Current Author Addresses: Ms. MacPherson; Drs. Hutchinson, Schneider, Awan, and Kimmelman; Ms. Oliviero; Ms. Feldhake; Ms. Ouimet; Mr. Sheng; and Ms. Wang: Biomedical Ethics Unit, Department of Social Studies of Medicine, McGill University, 3647 Peel Street, Montreal, QC H3A 1X1, Canada.

Dr. Papenburg: Department of Pediatrics, McGill University Health Centre, E05.1905, 1001 Décarie Boulevard, Montreal, QC H4A 3J1, Canada.

Dr. Basta: Department of Epidemiology, Biostatistics and Occupational Health, McGill University, 1020 Pine Avenue West, Montreal, QC H3A 1A2, Canada.
Author Contributions: Conception and design: A. MacPherson, C. Ouimet, J. Kimmelman.

Analysis and interpretation of the data: A. MacPherson, N. Hutchinson, O. Schneider, C. Wang, J. Papenburg, J. Kimmelman.

Drafting of the article: A. MacPherson, N. Hutchinson, $\mathrm{O}$. Schneider, E. Oliviero, J. Kimmelman.

Critical revision for important intellectual content: $\mathrm{N}$. Hutchinson, F. Awan, J. Papenburg, N.E. Basta, J. Kimmelman.

Final approval of the article: A. MacPherson, N. Hutchinson, $O$. Schneider, E. Oliviero, E. Feldhake, C. Ouimet, J. Sheng, F.A. Awan, C. Wang, J. Papenburg, N.E. Basta, J. Kimmelman.

Statistical expertise: N.E. Basta.

Obtaining of funding: A. MacPherson, J. Kimmelman.

Administrative, technical, or logistic support: A. MacPherson, F. A. Awan.

Collection and assembly of data: A. MacPherson, N. Hutchinson, O. Schneider, E. Oliviero, E. Feldhake, C. Ouimet, J. Sheng, F.A. Awan, C. Wang, J. Kimmelman.

\begin{tabular}{|c|c|c|c|c|c|}
\hline \multicolumn{6}{|c|}{ Appendix Table 1. Phase Transition Probabilities } \\
\hline Phase 1 to phase 2 & 15.5 & $38.2(30.7-45.0)$ & 76 & 1 & 143 \\
\hline Phase 3 to FDA approval & 14.1 & $61.1(3.7-84.3)$ & 9 & 1 & 15 \\
\hline
\end{tabular}

FDA $=$ U.S. Food and Drug Administration; POS = probability of success.

*Values shown are 1-Kaplan-Meier estimator $(95 \% \mathrm{Cl})$.

\begin{tabular}{|c|c|c|c|c|}
\hline \multicolumn{5}{|c|}{ Appendix Table 2. POS, by Vaccine Type, Sponsor Type, and Indication } \\
\hline Overall & $10.0(2.6-16.9)$ & 7 & 30 & 39 \\
\hline \multicolumn{5}{|l|}{ Vaccine type } \\
\hline Whole-pathogen & 0.0 (no variance) & 0 & 13 & 17 \\
\hline Split virus & $56.4(12.6-78.2)$ & 6 & 4 & 1 \\
\hline \multicolumn{5}{|l|}{ Sponsor type } \\
\hline Large company & $30.5(3.2-50.1)$ & 5 & 7 & 7 \\
\hline Foundation & 0.0 (no variance) & 0 & 4 & 3 \\
\hline \multicolumn{5}{|l|}{ Indication } \\
\hline Noninfluenza & $3.2(0.0-9.2)$ & 1 & 14 & 26 \\
\hline Influenza & $18.0(3.8-30.2)$ & 6 & 16 & 13 \\
\hline
\end{tabular}

POS = probability of success.

* Values shown are 1-Kaplan-Meier estimator $(95 \% \mathrm{Cl})$ at 10 years from phase 2 start. The set of trajectories included for each calculation may overlap, as some trajectories involved multiple vaccine and/or sponsor types.

† Three trajectories labeled "multiple" in Table 2 were included in the vaccine type analysis as both subunit and nucleic acid. The remaining 7 trajectories labeled "multiple" were not included in the analysis because they did not advance beyond phase 1. 


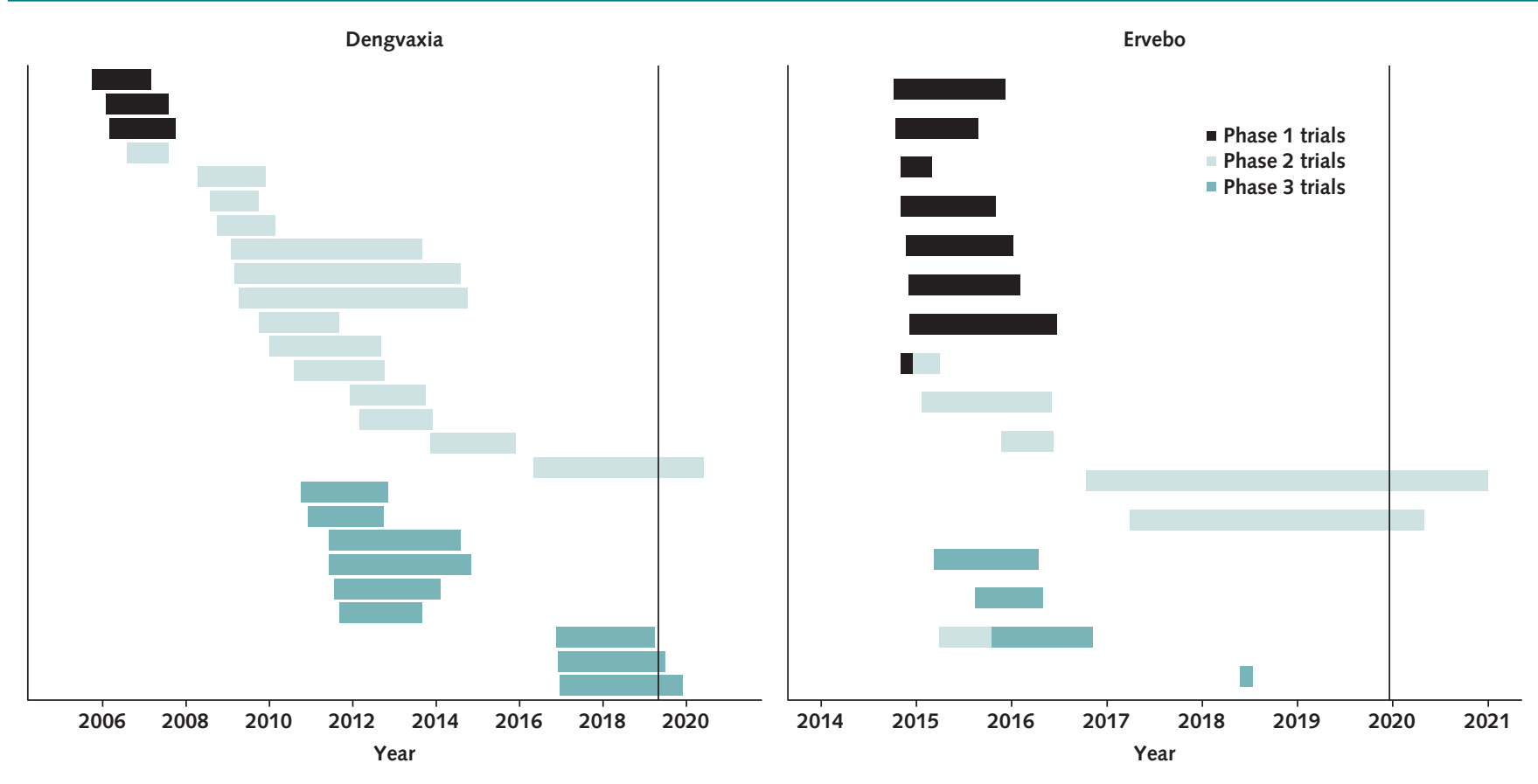

Each line represents a trial and spans the duration from trial start to primary completion date. Completed phase $1 / 2$ and phase $2 / 3$ trials are indicated by using both colors, with the first phase spanning a third of the total trial duration and the second phase spanning the remainder of the trial duration. The vertical black line indicates the U.S. Food and Drug Administration approval date.

Appendix Figure 2. Kaplan-Meier curves for the probability of transitioning from phase 1 to 2

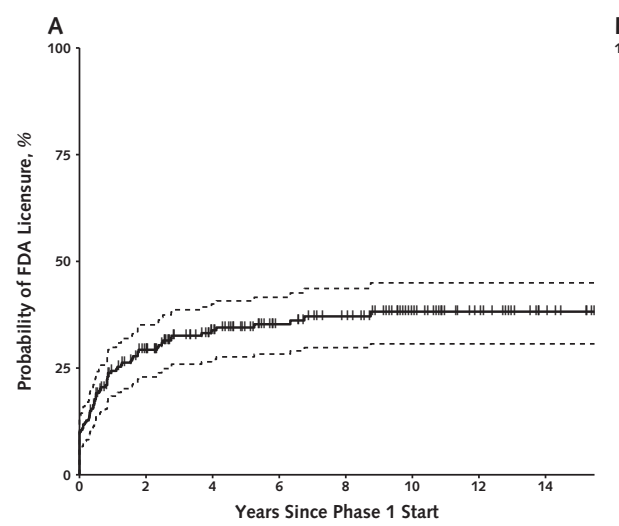

Vaccines with potential for approval, $n$

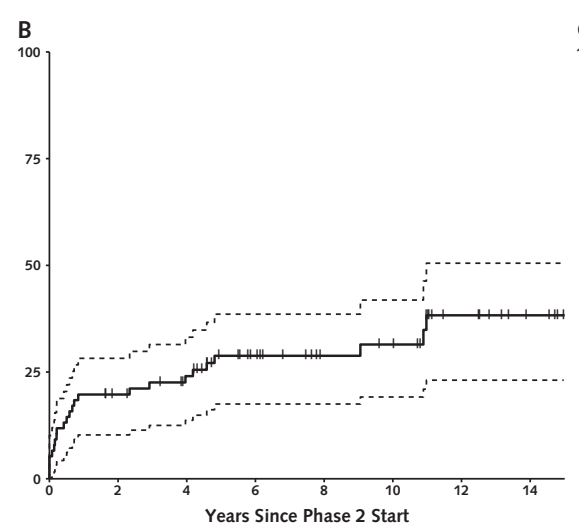

76

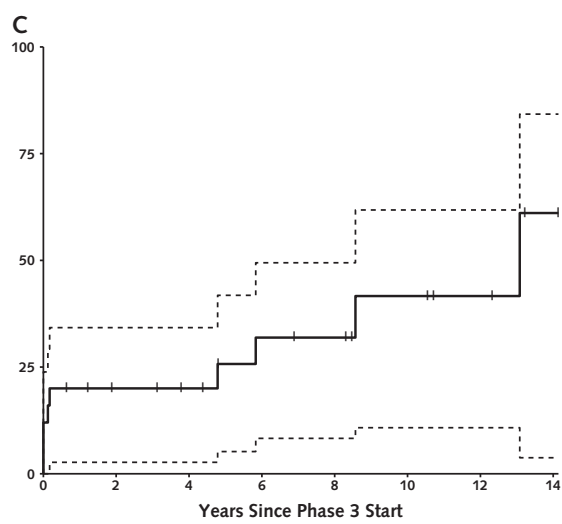

$(A)$, phase 2 to phase $3(B)$, and phase 3 to FDA licensure $(C)$ over time from trial launch.Vertical tick marks indicate censored vaccine trajectories. Dotted lines indicate $95 \%$ Cls. FDA = U.S. Food and Drug Administration. 